\title{
BMJ Open Observational study comparing the accuracy/variability between the ERSPC and the PCPT risk calculators for the prediction of significant prostate cancer in patients with PSA $<10 \mathrm{ng} / \mathrm{mL}$
}

\author{
Enrique Gomez Gomez, ${ }^{1,2}$ Juan José Salamanca Bustos, ${ }^{1}$ \\ Julia Carrasco Valiente, ${ }^{1,2}$ Jose Luis Fernandez Rueda, ${ }^{3}$ Ana Blanca, ${ }^{2}$ \\ José Valero Rosa, ${ }^{1,2}$ Ines Bravo Arrebola, ${ }^{1}$ Javier Marquez López, ${ }^{1}$ \\ Juan Manuel Jimenez Vacas, ${ }^{4}$ Raul Miguel Luque, ${ }^{4}$ Maria José Requena Tapia ${ }^{1,5}$
}

To cite: Gomez Gomez E, Salamanca Bustos JJ, Carrasco Valiente J, et al. Observational study comparing the accuracy/ variability between the ERSPC and the PCPT risk calculators for the prediction of significant prostate cancer in patients with PSA $<10 \mathrm{ng} / \mathrm{mL}$. BMJ Open 2019;9:e031032. doi:10.1136/ bmjopen-2019-031032

- Prepublication history and additional material for this paper are available online. To view these files, please visit the journal online (http://dx.doi. org/10.1136/bmjopen-2019031032).

Received 18 April 2019 Revised 04 October 2019 Accepted 04 October 2019

Check for updates

(c) Author(s) (or their employer(s)) 2019. Re-use permitted under CC BY-NC. No commercial re-use. See rights and permissions. Published by BMJ.

For numbered affiliations see end of article.

Correspondence to

Enrique Gomez Gomez;

enrique.gomez.gomez.sspa@ juntadeandalucia.es

Professor Raul Miguel Luque; raul.luque@uco.es

\section{ABSTRACT}

Introduction Risk calculators (RCs) are easy-to-use tools considering available clinical variables that could help to select those patients with risk of prostate cancer (PCa) who should undergo a prostate biopsy.

Objective To perform a comparison for the prediction of significant PCa (SigPCa) between the European Randomised Study of Screening for PCa (ERSPC) and the PCa Prevention Trial (PCPT) RCs in patients with prostatespecific antigen (PSA) between 3 and $10 \mathrm{ng} / \mathrm{mL}$ through an evaluation of the accuracy/variability between two consecutive PSA values.

Setting An observational study in a major university hospital in the south of Spain.

Methods and participants An observational study was performed in patients who underwent a prostate biopsy. SigPCa probabilities were calculated with the two PSA measures using ERSPC3/4+digital rectal examination and PCPT v2+free PSA RCs. The prediction of SigPCa was determined by the area under the receiver operating characteristic curve (AUC). Calibration, discrimination and decision curve analysis were studied. The variability between both RCs' agreement was compared using Cohen's kappa coefficient.

Results 510 patients were analysed (87 diagnosed with SigPCa). The median PSA values were 5.3 and $5 \mathrm{ng} / \mathrm{mL}$ for PSA1 and PSA2, respectively. Both RCs overestimated the risk in the case of high-risk probabilities. Discriminative ability for SigPCa was similar between models with an AUC $=0.73(0.68-0.79)$ for ERSPC-RC versus $0.73(0.67-$ 0.79 ) for PCPT-RC. ERSPC-RC showed less variability than PCPT-RC, with a constant agreement $(\mathrm{k}=0.7-0.8)$ for usual range of clinical decision-making. Remarkably, a higher number of biopsies would be avoided using the ERSPC$\mathrm{RC}$, but more SigPCa would be missed along all the risk probabilities.

Conclusions Both RCs performed similar in the prediction of SigPCa. However, ERSPC-RC seems to be more stable for intraindividual PSA variations.
Strengths and limitations of this study

- This study is the first to compare two available free risk calculators (RCs) in patients with a prostatespecific antigen (PSA) $<10 \mathrm{ng} / \mathrm{mL}$ analysing their variability between two consecutive different PSA levels.

- One of the study limitations is that prostate volume was an estimation and categorisation from a transrectal ultrasound (TRUS) calculation, and, therefore, it is not the actual approach for which the RC was developed.

- Although the clinical information of this study was extracted from a clinical practice cohort and with information that could be useful for urologists worldwide, this is a retrospective study and the use of TRUS biopsy for prostate cancer diagnosis, even though it is the standard in most populations, suffers from random error compared with template biopsy, which could have affected prediction results.

\section{INTRODUCTION}

Prostate cancer (PCa) is the second most frequently diagnosed malignancy in men worldwide and the most frequent in developed countries. ${ }^{1}$ Its current standard of diagnosis is a prostate biopsy based on prostate-specific antigen (PSA) levels and digital rectal examination (DRE). However, there are other available and complementary variables that could help to select those patients who should undergo a prostate biopsy (such as age, prostate volume, free PSA, family history, etc), but these are not always used and/or well integrated in daily clinical practice. ${ }^{2}$ In line with this, risk calculators (RCs) are easy-to-use tools that can help the clinicians to take advantage of all these 
available variables. ${ }^{3}$ The two main RCs are from the European Randomised Study for Screening of Prostate Cancer (ERSPC cohort; ERSPC-RC: http://www.prostatecancerriskcalculator.com/seven-prostate-cancer-riskcalculators) and from the Prostate Cancer Prevention Trial (PCPT cohort; PCPT-RC: http://deb.uthscsa.edu/URORiskCalc/Pages/calcs.jsp). Both RCs have undergone some modifications, specifically the addition of estimated prostate volume in the ERSPC-RC. ${ }^{45}$ Furthermore, both RCs were originally developed from different patient cohorts and each RC uses different variables.

To date, limited external validations and comparisons have been performed by different groups. ${ }^{6-9}$ The two most important recent comparisons of the modified RCs were performed by Foley et $a l^{67}$ and Poyet $e t a l^{9}$ Both found a better discriminative ability for ERSPC-RC versus PCPT v2-RC for the diagnoses of significant PCa ( $\mathrm{SigPCa}$ ) (area under the receiver operating characteristic curve (AUC) around 0.74 vs 0.69 , respectively), but they also included patients with a high PSA of up to $50 \mathrm{ng} / \mathrm{mL}$. Despite the possibility of using these RCs in patients with PSA levels up to $50 \mathrm{ng} / \mathrm{mL}$, it is clear that the advantages of using both RCs would probably increase in patients with a PSA under $10 \mathrm{ng} / \mathrm{mL}$, where the rate of positive biopsy for PCa clearly decreases, with an important number of unnecessary biopsies. Furthermore, in the case of the PCPT v2-RC, the addition of the free PSA value in patients with a PSA under $10 \mathrm{ng} / \mathrm{mL}$ seems to improve its predictive ability, ${ }^{5}$ and, therefore, given its accessibility, this value should be included in the RC.

The intraindividual and interassay variability of PSA is already known ${ }^{10-12}$ and, therefore, at least two measures are necessary before a prostate biopsy is indicated. In fact, it has been shown that approximately $25 \%$ of men with initial PSA levels between 4 and $10 \mathrm{ng} / \mathrm{mL}$ had normal PSA values on repeat testing. ${ }^{13}$ In line with this, despite being primarily based on PSA level, the variability of the two RCs mentioned above has been poorly studied and might have implications for patient management. Our group has recently evaluated this variability with the ERSPC-RC, which showed stable accuracy over a cohort of patients, but some changes with respect to an individual approach. ${ }^{14}$ To date, there is no study comparing the accuracy and variability of both RCs, the ERSPC+DRE versus the PCPT v2+free PSA, for the prediction of SigPCa. Therefore, the aim of this study was to perform a direct comparison between ERSPC+DRE and PCPT v2+free PSA RCs in patients with a PSA between 3 and $10 \mathrm{ng} / \mathrm{mL}$, evaluating the accuracy and variability of both methods in the prediction of SigPCa.

\section{MATERIALS AND METHODS}

\section{Study population and design}

An observational retrospective study was performed in patients from ONCOVER cohort (1021 biopsies indicated by clinical practice wherein patients donated blood and urine before the biopsy).
Blood sample was obtained in the morning (between 8:00 and 10:00) after fasting overnight, and then the prostate biopsy was implemented according to clinical practice. The inclusion criteria for this study were (1) PSA indication between 3 and $10 \mathrm{ng} / \mathrm{mL}$, (2) full clinical and laboratory data to fulfil ERSPC-RC and PCPT-RC criteria, (3) age 55-80 years and (4) two consecutive measurements of PSA levels within an interval of 12 weeks. Exclusion criteria included patients with a previously known PCa diagnosis or treatment that could modify PSA levels (online supplementary table 1 ).

Transrectal prostate biopsy was carried out under local anaesthesia by using a standard periprostatic block, a transrectal ultrasound (TRUS) transducer and an 18G automated needle biopsy instrument. The prostatic volume was measured following the protocol used during TRUS, and usual recommendations were to take 12 cores in patients undergoing the first biopsy procedure and a minimum of 16 biopsy cores for those who had a previous biopsy. Biopsy specimens were analysed by expert urological pathologists according to the International Society of Urological Pathology 2005 modified criteria. ${ }^{15}$

\section{Main variables description}

Demographic information and the medical history of each patient were obtained. PSA levels were measured twice within a period no longer than 12 weeks, as follows: (1) PSA1 and free PSA1, for biopsy indication; and (2) PSA2 and free PSA2, before undergoing prostate biopsy. Both PSA measures were evaluated by chemiluminescent microparticle immunoassays (ng/mL, Ref. 7k70; Abbott). The median and IQR of time between measurements was 6 (3-8) weeks.

Prostate volume: estimated by TRUS and categorised in three possible values, $25-40-60 \mathrm{~mL}$, as recommended ${ }^{4}$ (TRUS volume $<30=25 \mathrm{cc}, 30-50=40 \mathrm{cc}, \geq 50=60 \mathrm{cc}$ ).

Significant/high-grade (HG) prostate cancer (SigPCa): PCa with a Gleason grade $\geq 7$ on biopsy.

\section{ERSPC-RC and PCPT-RC probabilities calculation}

ERSPC: The formulas for the ERSPC-RC3+DRE for patients at initial biopsy and the ERSPC-RC4+DRE for patients at repeat biopsy were used in this study. These calculators use PSA, prostate volume and DRE as variables, with a negative prostate biopsy in ERSPC4+DRE in patients who had a previous biopsy. This provides a probability rating for any PCa or SigPCa (Gleason $\geq 7$ ).

ERSPC1/SigPCa (first measure): risk probability calculated by ERSPC-RC3 or 4 (if previous biopsy)+DRE; used PSA1 to calculate the predicted risk for both any PCa and SigPCa (HG PCa).

ERSPC2/SigPCa (second measure): risk probability calculated by ERSPC-RC3 or 4 (if previous biopsy)+DRE; used PSA2 to calculate the predicted risk for both any PCa and SigPCa (HG PCa).

PCPT: The formula for the PCPT-RC $2.0+\%$ free PSA was used in this study. This calculator uses race, age, PSA level, \%free PSA level, family history of PCa, DRE and 
prior prostate biopsy. This gives a probability of negative biopsy, low-grade PCa and SigPCa (Gleason $\geq 7$ ).

PCPT 1: Risk probabilities calculated by PCPT $2.0+\%$ free PSA using PSA1 and free PSA1.

PCPT2: Risk probabilities calculated by PCPT $2.0+\%$ free PSA using PSA2 and free PSA 2.

The variability of PSA was calculated by the following formula: Measure 1-Measure 2I/Measure 1.

\section{Statistical analysis}

A descriptive study was performed by calculating the median and IQRs for the quantitative variables, and the absolute frequencies and percentages for the qualitative variables. Student's t-test for paired groups was used to compare the means of the quantitative variables (PSA1 and 2).

The investigation of the comparative performance in the detection of SigPCa of both RCs, ERSPC-RC and PCPT-RC, was performed, taking into account these four factors: discrimination capacity, calibration, clinical utility and consistency against the observed variations in PSA levels for our dataset.

The discriminative ability of the models, that is, their ability to separate those patients who had SigPCa from those who do not, was assessed using the area under the receiver operating characteristic (ROC) curve (AUC), ${ }^{16}$ as measured in our sample. This is one of the most frequently used measurements of model discrimination because of its independence of the selection of a specific decision threshold and its robustness against class imbalance. CIs for these AUCs were computed using bootstrapping. These AUCs were then compared to determine the relative performance of the models using DeLong tests. ${ }^{17}$ These tests were chosen because of their non-parametric nature, with few assumptions about the data, and their suitability for paired data, as both models were evaluated over the same dataset, properties which make this the most commonly used test to compare AUCs. ${ }^{18}$ For this comparison, we focused on the calculated risk score using the first measure of PSA (PSA1; the value which met the criteria for a prostate biopsy).

The calibration of the calculators for our cohort was then investigated to determine the agreement between the frequency of the observed outcome (SigPCa in our case) and the risks predicted by the model. Calibration plots were used for this purpose ${ }^{19}$ enabling a visual evaluation of this agreement and the comparison between RCs.

To address the potential clinical utility of the models, we performed decision curve analysis on our data, as proposed by Vickers and Elkin. ${ }^{20}$ This method has the advantage of not requiring the specification of the relative cost for false positives and false negatives, defining a net benefit as a function of the decision threshold at which one would consider obtaining a biopsy.

Finally, the stability of the predictions of both RCs, with regard to the observed intrapatient changes on PSA levels between measurements, was investigated using Cohen's kappa ( $\kappa)$ inter-rater agreement coefficient as a function of the decision threshold. This coefficient was selected due to its widespread use and robustness against random agreements and, thus, is a better measurement than naïve accuracy.

All the analyses were performed using SPSS V.17.0 and R V.3.2.3 (R Foundation for Statistical Computing, Vienna, Austria; https://www.R-project.org/). A $<5 \%$ level of significance $(p<0.05)$ was used to decide statistically significant differences.

\section{Patient and public involvement}

Participants and public were not involved in the development of research questions, study design or recruitment.

\section{RESULTS}

\section{Cohort characteristics}

In the present study, we analysed 510 patients who met the inclusion criteria previously described. Median age was $65(60-70)$ years, with a family history in 89 patients $(17.5 \%)$ and a suspicious DRE in 82 patients $(16.1 \%)$. The median PSA before prostate biopsy indication was 5.3 (4.3-6.9) ng/mL. A total of 176 patients were diagnosed with PCa, 87 of those categorised as SigPCa. Most patients $(\mathrm{n}=401 ; 78.6 \%)$ were biopsy naive and the median prostate volume was 35 (26-49) cc. Further cohort description according to SigPCa status is shown in table 1 (and in online supplementary data).

In total, 66 patients had a PSA2 out of the range of 3-10 $\mathrm{ng} / \mathrm{mL}$ due to the variability ( 50 patients below $3 \mathrm{ng} / \mathrm{mL}$ and 16 patients above $10 \mathrm{ng} / \mathrm{mL}$ ); thus, in this case, the $\%$ free PSA was not calculated, and the risk probability was calculated without the inclusion of this variable. The patients were maintained in the analysis as reflecting the variability of the PSA, and the application of the models in this real situation, although acknowledging that it could introduce a bias in terms of calibration and variability.

\section{Direct comparison for SigPCa prediction}

Discrimination ability for SigPCa was no different between the two models (ERSPC1-RC vs PCPT1-RC: 0.73, 95\% CI 0.68 to 0.79 vs 0.73 , $95 \%$ CI 0.67 to 0.79 , respectively). ROC curves are shown in figure 1A. Similarly, no difference was found in the discrimination ability for any PCa. The comparison of the RC for both measures is described in figure 1B-D with similar results but a tendency of better accuracy for PCPT2-RC versus ERSPC2-RC $(\mathrm{p}=0.25)$. Online supplementary table 2 shows multiple comparisons by the DeLong test resulting in no differences between the two RCs for SigPCa.

Both models tended to overestimate the risk for a high probability of SigPCa, and slightly underestimate it for low-risk patients, suggesting that the models would benefit from a recalibration for our population (figure 2). None of the models predicted very high probabilities for most patients. The calibration curves for any PCa are shown in online supplementary figure 1 . 
Table 1 Clinical and demographic characteristics of the cohort of patients categorised according to cancer status

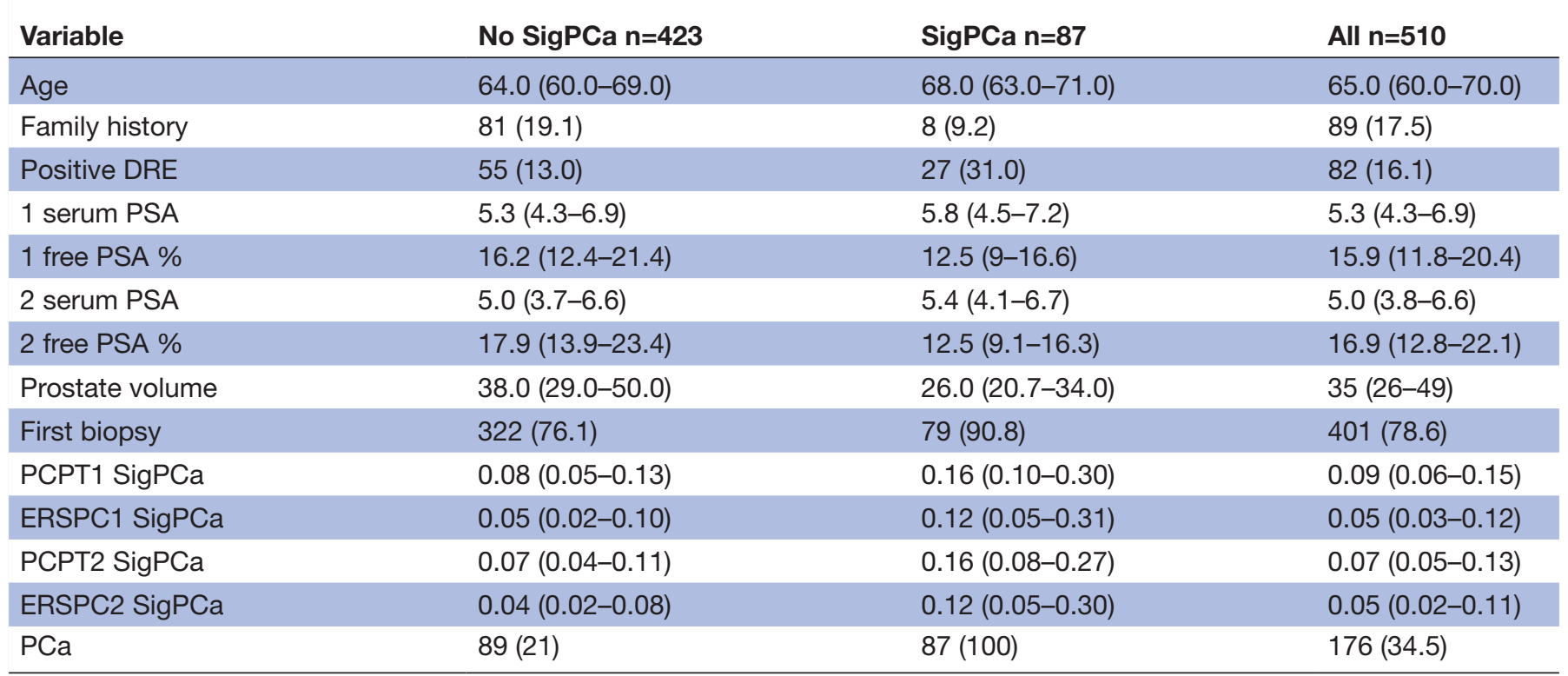

Median values (IQR) are expressed for quantitative variables and absolute values (percentage) for qualitative variables.

DRE, digital rectal examination; ERSPC1/PCPT1 SigPCa, probability of high-grade PCa using the first measurement of serum PSA (at the time of biopsy indication by the urologist); ERSPC2/PCPT2 SigPCa, probability of high-grade PCa using the second measurement of serum PSA (just before undergoing prostate biopsy); No SigPCa, no cancer or non-significant PCa; PCa, prostate cancer; PSA, prostate-specific antigen; SigPCa, significant PCa (Gleason $\geq 7$ on biopsy).

The decision curve analyses revealed that both RCs provided a clinical net benefit in the threshold probability range for SigPCa (figure 3). The net benefit was comparable between the two RCs for SigPCa.

As shown in online supplementary figure 2, the addition of free PSA clearly improved the discriminative ability of the PCPT-RC (0.65 (0.59-0.71) PCPT1 v2.0-RC vs $0.73(0.67-0.79)$ PCPT1 v2.0+free PSA-RC; $\mathrm{p}=0.02)$.

\section{Variability and clinical significance}

PSA and free PSA change was significantly different between the two measures, but with low clinical variations (average PSA1 $5.69 \mathrm{ng} / \mathrm{mL}$ vs PSA2 $5.39 \mathrm{ng} / \mathrm{mL}(\mathrm{p}<0.05)$ and average free PSA1 $16.99 \%$ vs free PSA2 $18.03 \%$ $(\mathrm{p}<0.05))$. Median variability of PSA was $14 \%(6 \%-27 \%)$. Taking into account this variability of PSA, ERSPC proved to be more stable than PCPT. The $\kappa$ agreement between ERSPC1 and ERSPC2 was practically constant, $0.79 \pm 0.09$ for the usual range of clinical decision $(0-0.3)$. However, PCPT1 and PCPT2 showed wider variations, with a $\kappa$ agreement of approximately $0.55 \pm 0.32$ in the same range, with a subsequent rapid decrease. The agreement between both models (ERSPC1 vs PCPT1) proved to be worse for thresholds in this range, peaking 0.47 for a $17 \%$ risk, with an average $0.32 \pm 0.12$ on the interval. The comparison between ERSPC2 and PCPT2 yielded similar results (figure 4).

Direct comparison of sensitivity and specificity of both RCs along the different clinical risk thresholds showed that PCPT-RC has higher sensitivity and lower specificity than ERPSC-RC for a given threshold along the clinically useful region (figure 5). The balance point is reached at a different risk threshold for each RC. The performances of both RCs at this point are comparable, as shown in figure 5. Considering the superposition of their respective ROC curves to a good approximation (figure 1), this means that a transformation of decision thresholds can make both models perform similarly.

\section{DISCUSSION}

Currently, considerable research is being carried out to find new diagnostic markers for SigPCa in order to reduce the number of biopsies and the overdiagnosis of insignificant $\mathrm{PCa} .{ }^{21}$ These markers are based on body fluids (blood, urine) or image explorations. ${ }^{22}{ }^{23}$ Some are recommended by guidelines such as the $4 \mathrm{k}$ score test, PCA3 and/or the Prostate Health Index (PHI) in body fluids (only PCA3 and PHI have been approved by the FDA), ${ }^{24-26}$ or multiparametric MRI (mpMRI), with recent evidence of its advantages in biopsy-naïve patients. ${ }^{27}$ However, costs and availability minimise their implementation worldwide, and, therefore, it is clear that additional and readily available tools, such as RCs, should be implemented in daily clinical practice. The two most used RCs are ERSPC-RC and PCPT-RC, which have been modified and adapted. ${ }^{45}$ Few external validations have been conducted, with varying results. ${ }^{79} 28$ Usually, external validations of RCs show worse performance than the original validations, ${ }^{8}$ a fact that is corroborated by our study. 
A

High-grade tumour

ERSPC1 vs PCPT1

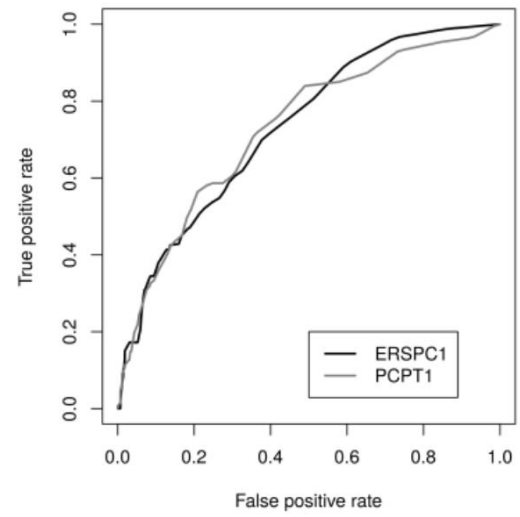

\begin{tabular}{lc}
\hline Risk Calculator for Sig PCa & AUC $\left(\mathbf{C l}_{95 \%}\right)$ \\
\hline ERSPC1 & $0.73(0.68,0.79)$ \\
PCPT1 & $0.73(0.67,0.79)$
\end{tabular}
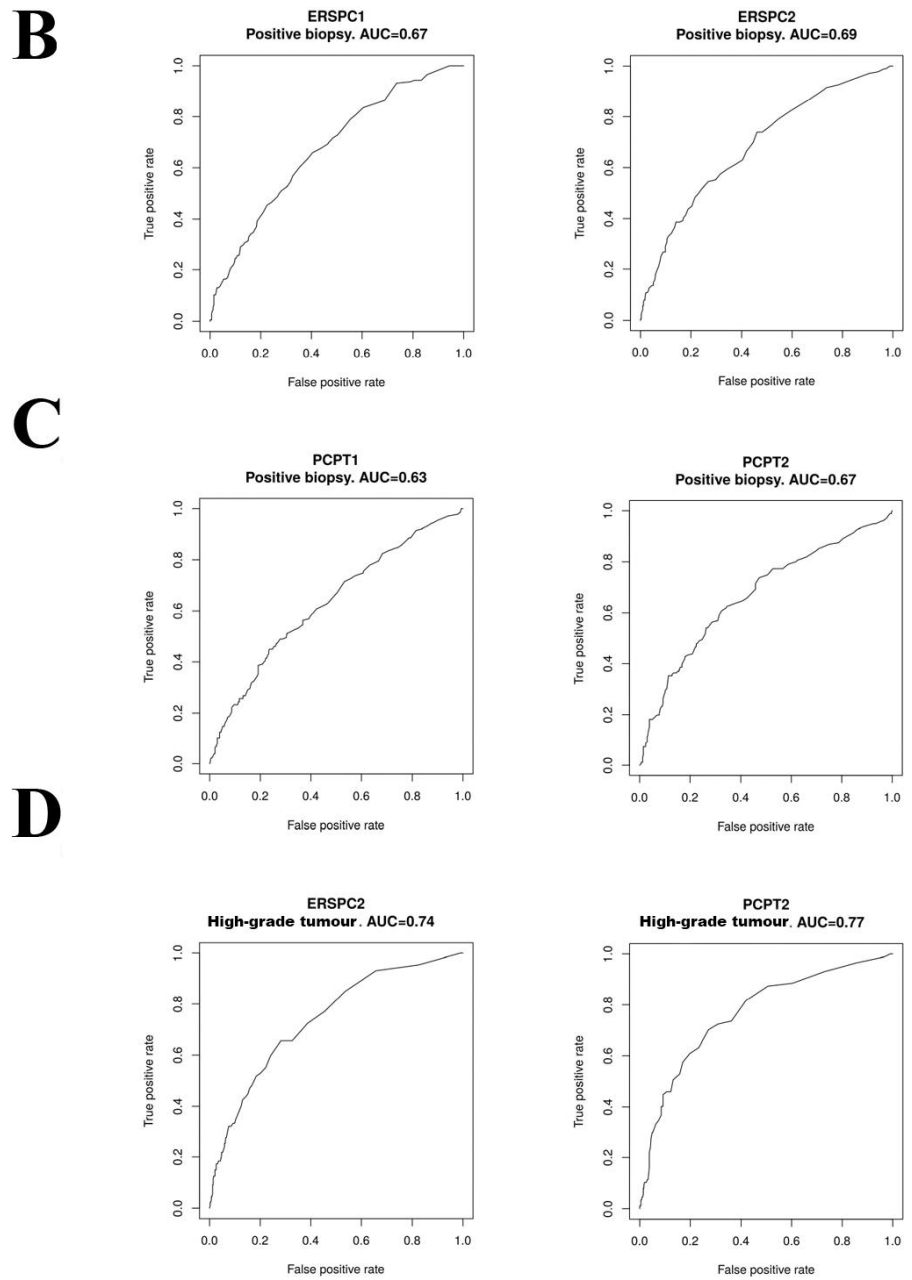

\begin{tabular}{lcc} 
AUC (Cl $\left.{ }_{95 \%}\right)$ & PB & HG \\
\hline ERSPC1 & $0.67(0.63,0.72)$ & $0.73(0.68,0.79)$ \\
ERSPC2 & $0.69(0.64,0.73)$ & $0.74(0.68,0.80)$ \\
PCPT1 & $0.63(0.58,0.68)$ & $0.73(0.67,0.79)$ \\
PCPT2 & $0.67(0.62,0.72)$ & $0.77(0.71,0.82)$
\end{tabular}

Figure 1 Receiver operating characteristic curves and area under the curve (AUC) values: (A) for the ERSPC1-RC (black) and PCPT1-RC (grey) for SigPCa; (B) for the ERSPC1-RC and the ERSPC2-RC for positive biopsy; (C) for the PCPT1-RC and the PCPT2-RC for positive biopsy; (D) for the ERSPC2-RC and the PCPT2-RC for SigPCa. HG, high grade; PB, positive biopsy. 
A

High grade tumour: ERSPC1

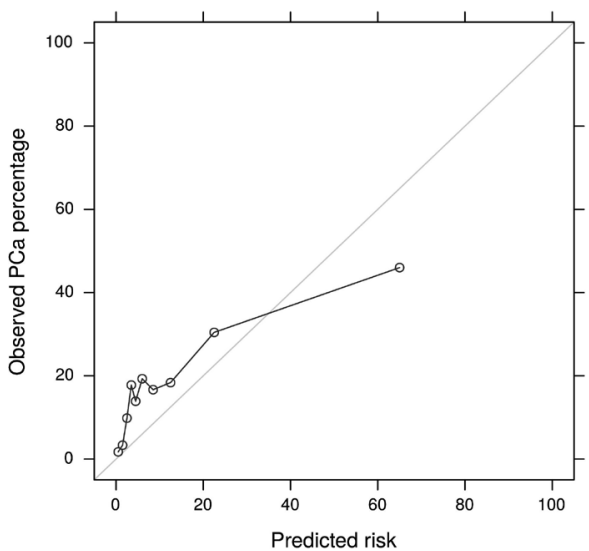

B

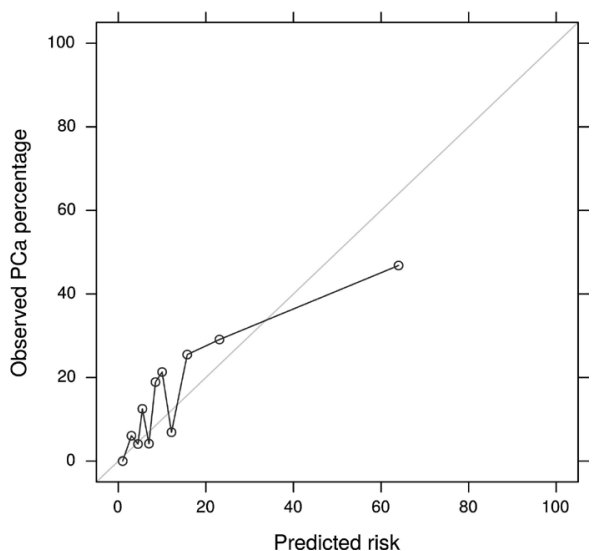

Figure 2 Calibration plots for risk estimation, showing the agreement between predicted risk (horizontal axis) and the actual observed prevalence for people with that risk (vertical axis). The diagonal line shows the ideal behaviour of a perfectly calibrated risk calculator, separating the upper left region where risks are underestimated from the lower right, where they are overestimated. (A) Calibration plots for ERSPC1-RC SigPCa risk estimation. (B) Calibration plots for PCPT1-RC SigPCa risk estimation. PCa, prostate cancer; SigPCa, significant PCa.

Therefore, based on all this information, evaluations, validations and incorporation of RCs are needed. ${ }^{3}$

The present study explores and compares for the first time both the PCPT v2+free PSA and the ERSPC+DRE for accuracy and also for variability and clinical relevance. Our group previously explored the accuracy and variability of the ERSPC+DRE RC, ${ }^{14}$ but in this study, we have specifically focused only on patients in the grey zone (PSA 3-10 ng/mL) and compared the ERSPC+DRE RC versus the PCPT v2+free PSA, an analysis that has not been previously performed. This comparison showed that both RCs had similar accuracy for the discrimination of SigPCa. However, ERSPC-RC had better calibration and stability for intraindividual PSA variations. Our methodology in

A

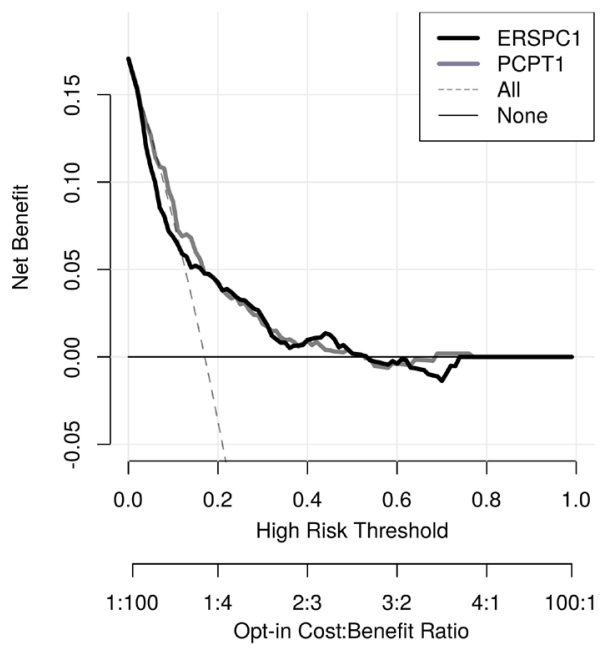

calculating the volume is an estimation from the results of the TRUS measure, similar to Poyet $e t a l$, and following the recommendations of Roobol et al. ${ }^{4}$ We have focused only on those patients with PSA between 3 and $10 \mathrm{ng} /$ $\mathrm{mL}$ who require additional diagnostic information. The PCPT-RC option with free PSA, which increases the accuracy of discrimination between SigPCa and no $\mathrm{PCa},{ }^{5}$ was calculated, as it is an easy-to-use and readily available tool for these patients.

As defined in the methodology, the first measure (ERSPC1 and PCPT1) was the focus of the direct comparison, as this was used as the indication for biopsy. The accuracy of both RCs was similar for SigPCa in our study, showing an accuracy similar to other external validations

B

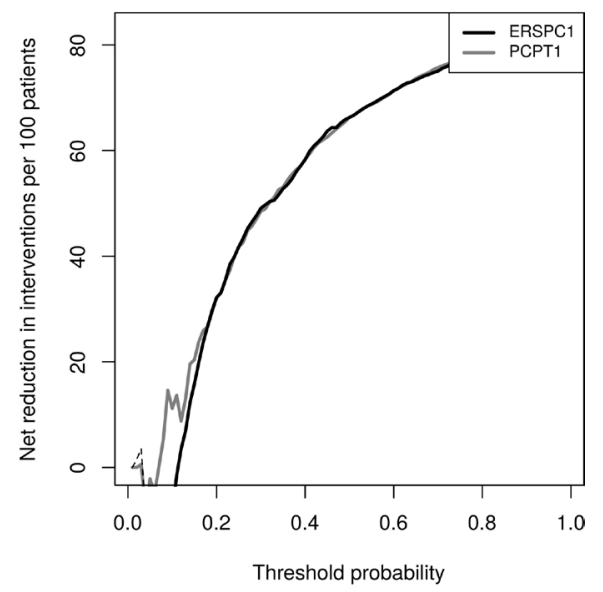

Figure 3 Results of the decision curve analysis. (A) Net benefit for the prediction of SigPCa on biopsy using the ERSPC1-RC (black line) and the PCPT1-RC (grey line) as a function of the risk threshold, compared with those benefits of the strategies of treating all patients (dashed line) and treating none (thin line). (B) Plot demonstrating net reduction of interventions per 100 patients using the ERSPC-RC (black line) and the PCPT-RC (grey line). ERSPC, European -Randomised Study of Screening for PCa; PCPT, PCa Prevention- Trial. 

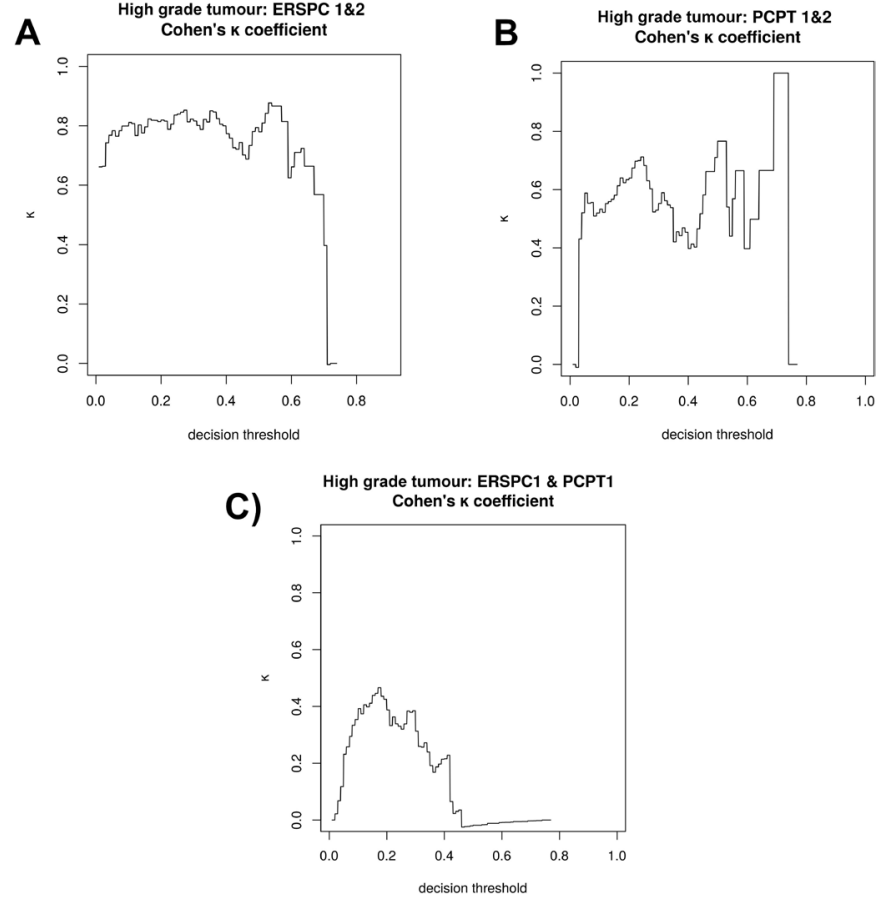

Figure 4 Graphics showing Cohen's $\kappa$ coefficient, which evaluated the agreement between risk calculators, as a function of the decision threshold, with 1 being total agreement and 0 being the worst possible expected agreement between rates. (A) Agreement between ERSPC1$\mathrm{RC}$ and ERSPC2-RC for significant prostate cancer (SigPCa). (B) Agreement between PCPT1-RC and PCPT2-RC for SigPCa. (C) Agreement between ERSPC1-RC and PCPT1-RC for SigPCa.

such as Poyet $e t a t^{\dagger}$ and Foley $e t a l^{7}$ for ESRPC (AUC $=0.73$ and 0.74 , respectively) and a better accuracy for PCPT1 v2. 0 when adding free PSA (AUC $=0.70$ and 0.69 , respectively). Still, these results are far from ideal, and thus, additional data from imaging or fluid markers might be included to improve the accuracy of the RCs. In agreement with the accuracy results, the decision curve analysis was also similar between both RCs. In fact, both RCs showed a net benefit from an early risk threshold, which means that their implementation would be useful in the pathway of patient selection.

Studying the variability of the RCs improves our knowledge about their stability, which could translate into improved decision-making and selection of patients. Our PSA cohort showed a variability that was in the range of that previously shown in the literature. ${ }^{11229}$ Our group and others ${ }^{1430}$ have demonstrated that a higher PSA variability is associated with a reduced risk of SigPCa in a prostate biopsy, but it does not improve the accuracy of a RC. However, probability stability is important in order to trust RC probabilities at any point. Our study shows good agreement between the two ERSPC+DRE RC probabilities, with good calibration and stability despite intraindividual PSA variations. PCPT v2+free PSA shows worse stability and higher variability, which could be explained simply by the fact that it uses two values (PSA and free PSA) that

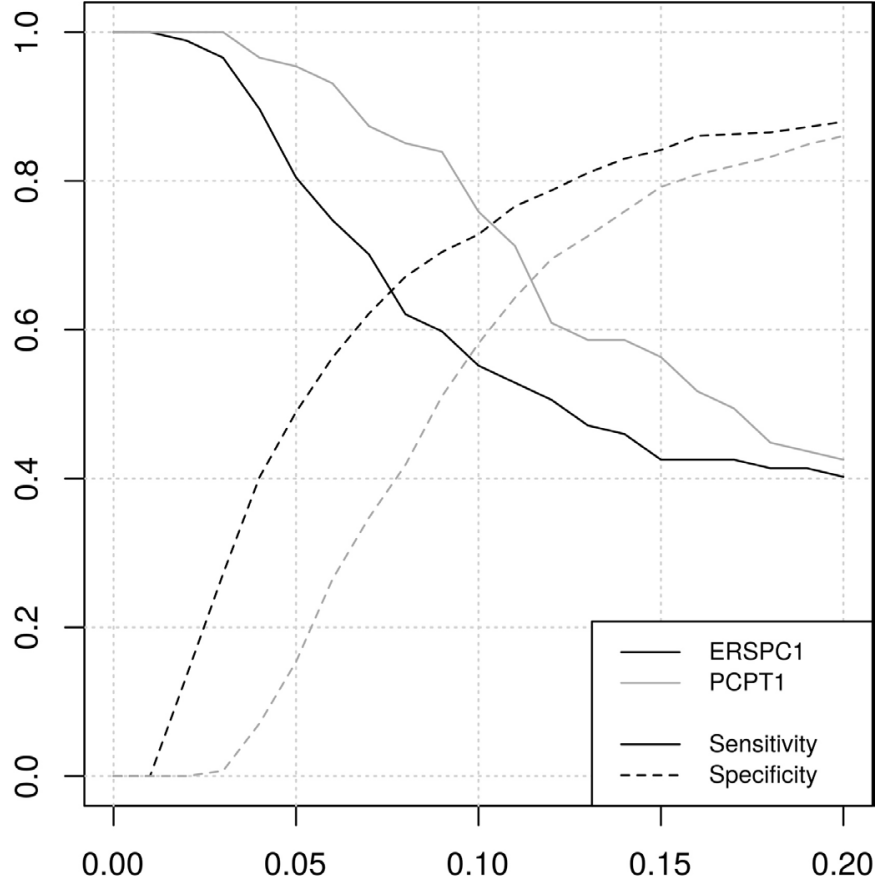

risk threshold

Figure 5 Graphics showing sensitivities and specificities of both risk calculators along the clinically useful risk threshold. The ERSPC-RC (black line) and the PCPT-RC (grey line). ERSPC, European -Randomised Study of Screening for PCa; PCPT, PCa Prevention- Trial.

suffer from this variability, ${ }^{31}$ while the use of an estimated volume in the ERSPC dilutes the PSA variability. These results should be interpreted with caution, as volume estimation was performed by categorisation of TRUS and not by DRE. It is true that this categorisation has previously shown good correlation. ${ }^{4}$ This likely depends on prostate volume $^{32}$ as well as low but certain interexaminer variability ${ }^{33}$ which could also increase ERSPC variability in an interclinician comparison. It should also be taken into account that the clinical translation of this stability is not clear, first, because of the limitation of the use of a single estimated prostate volume and because the global accuracy of both RCs are not significantly different, and seems to have a tendency to improve in the PCPT2 RC.

Calibration plots show that both models (PCPT-RC and ERSPC-RC) predict adequately only the actual risk of PCa and SigPCa for low-risk patients, with a wider useful range in the case of $\mathrm{PCa}$ and a lower range in the case of SigPCa. For higher risk patients, the calibration curves become irregular. This effect is accentuated for risks close to 1 , as both models predict maximum risks of around 0.75 for SigPCa. The models would benefit from recalibration for our population in the low-moderate risk region, considering that this is the region of greater interest for the model, as patients with a high predicted risk would probably undergo biopsy anyway. Nonetheless, despite not showing a good calibration in the usual range for clinical decision (0-0.3), visually ERSPC seems to be more consistent with a less fluctuating calibration in this 
range compared with PCPT, but at this point, this should be confirmed in future studies because no conclusion for direct comparison about calibration could be reached in the present study as quantitative analysis is outside the aim of this research. The comparison of coefficients between PCPT1 and PCPT2 and between PCPT2 and ERSPC2 showed that the differences between PCPT1 and PCPT2 were similar to those between ERSPC and PCPT models. As previously discussed, ERSPC seems to be more insensitive and, therefore, robust to intraindividual variations of PSA compared with PCPT, while the predictive performance is similar and the clinical translation not clear yet.

Despite the similar decision curve, results from the sensitivity, specificity and ROC curve analysis show that the same risk threshold should not be used for both models. Both RCs are able to have similar performance, and the benefit of using any of them is similar in order to screen patients for a prostate biopsy, if the correct cut-off point is selected. It should be highlighted the importance of having an almost $100 \%$ negative predictive value, as the advantage of reducing unnecessary biopsies should not be at the cost of missing or delaying the diagnoses of a SigPCa.

In clinical practice, the use of these RCs should be the first step in guiding the decision for further management of the patient. Patients with a confirmed, elevated PSA between 3 and $10 \mathrm{ng} / \mathrm{mL}$ should be better stratified using other variables within a RC, as men with PSA levels $>10$ $\mathrm{ng} / \mathrm{mL}$ are likely to proceed to biopsy regardless of other factors. Probably a specific cut-off point in the risk probability should not be used and take advantage of the known probabilities to discuss with the patient the biopsy indication as recommended by the PCPT-RC. In the situation in which the patient is in the low-risk group, according to both RCs (ERSPC and PCPT), the patient could continue with just follow-up. This fact has also been proposed by Alberts $e t a l^{34}$ when applying new diagnostic markers, such as mpMRI. Specifically, they showed that following a negative recommendation from the ERSPC-RC would have avoided $62(51 \%)$ of 122 mpMRIs and two $(25 \%)$ of eight insignificant PCa diagnoses, missing three $(10 \%)$ of $31 \mathrm{HG}$ PCa. As the positive predictive value of these RCs is not as good as their negative predictive value, in case of discordance between both RCs or if there is an indication for a biopsy according to both RCs, other images or fluid biomarkers could increase the accuracy in order to potentially reduce the harm from unnecessary prostate biopsy and overdiagnosis. ${ }^{35}$ Specifically, Loeb et $a l^{36}$ has recently demonstrated that the incorporation of PHI into both RCs increases the accuracy of the diagnoses of SigPCa. Another relevant point should be comment from the tendency of better predictive ability with the second evaluations of PSA, reinforcing the idea of the need of several PSA values to confirm the risk and discarded confounding factors. Furthermore, this analysis could suggest a tend towards better discrimination ability of PCPT in the range of lower probabilities (when PSA is low), but further research would be needed to validate this hypothesis. These RCs only show a static probability, so other longitudinal variables and clinical judgement should be required for their application.

The present study has some limitations. First, despite the prospectively collected information, it is a retrospective study design. Second, prostate volume was an estimation and categorisation from a TRUS calculation, and, therefore, it is not the actual approach for which the RC was developed. Third, the PSA values interval was not the same for all patients, which means the results should be interpreted with caution. Fourth, the use of TRUS biopsy for PCa diagnosis, although it is the standard in most populations, suffers from random error compared with template biopsy ${ }^{37}$ which could have affected prediction results. However, the clinical information was extracted from a clinical practice cohort and with information that could be useful for urologists worldwide.

Altogether, our results showed that (1) the use of both RCs (ERSPC and PCPT) could be a useful tool in the selection of patients who need prostate biopsy, and that both RCs performed similar in the prediction of SigPCa; (2) ERSPC-RC showed higher stability than PCPT-RC for intraindividual PSA variations; and (3) when comparing both RCs' sensitivity and specificity, a higher rate of biopsies could be avoided with the ERSPC-RC versus the PCPT-RC, but with a higher rate of SigPCa missed. Thus, in those patients with a PSA between 3 and $10 \mathrm{ng} / \mathrm{mL}$, these tools should be used in order to improve selection and specificity. The RCs specifically should be selected according to the variables available in the clinic. In addition, both RCs could also be used and the decision to undergo a biopsy be shared with the patient.

\section{Author affiliations}

${ }^{1}$ Urology, Hospital Universitario Reina Sofia, Cordoba, Spain

${ }^{2}$ Genitourinary Diseases, Maimonides Institute for Biomedical Research of Cordoba,

Cordoba, Spain

${ }^{3}$ Innovation and Methodology, Maimonides Institute for Biomedical Research of Cordoba, Cordoba, Spain

${ }^{4}$ Oncobesity and Metabolism, Maimonides Institute for Biomedical Research of Cordoba, Cordoba, Spain

${ }^{5}$ Instituto Maimónides de Investigación Biomédica de Córdoba (IMIBIC), Hospital Universitario Reina Sofía, Universidad de Córdoba, Cordoba, Spain

\section{Twitter Enrique Gomez Gomez @enriquegomezgo4}

Contributors EGG, RML, MJRT and JCV carried out the conception and design of the study. EGG, JJSB, JCV, AB, JVR, IBA, JML and JMJV contributed to the data acquisition. EGG, JCV, JLFR, RML and MJRT carried out the analysis and interpretation of data. EGG, JCV and RML drafted the manuscript. JJSB, JLFR,

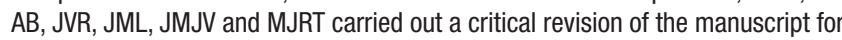
important intellectual content. EGG and JLFR performed the statistical analysis. RML, MJRT and JCV supervised the work.

Funding This work was funded by the Spanish Ministerio de Economía y Competitividad (MINECO) and FEDER programme (Projects 'Development of methods for early cancer detection', (CCB.030PM)), Instituto de Salud Carlos III and co-funded by European Union (ERDF/ESF, 'Investing in your future': PI16/00264, CM16/00180) and CIBERobn. CIBER is an initiative of Instituto de Salud Carlos III, Ministerio de Sanidad, Servicios Sociales e Igualdad, Spain. This work has been awarded by the Real Academia de Medicina de Sevilla 2018. The funding agreement ensured the authors' independence in designing the study, interpreting the data, writing and publishing the report.

Competing interests None declared. 
Patient consent for publication Obtained.

Ethics approval This study was performed as part of the ONCOVER project. Ethical approval was obtained by the Reina Sofia Hospital Research Ethics Committee in accordance with the Declaration of Helsinki.

Provenance and peer review Not commissioned; externally peer reviewed.

Data availability statement All data relevant to the study are included in the article or uploaded as online supplementary information.

Open access This is an open access article distributed in accordance with the Creative Commons Attribution Non Commercial (CC BY-NC 4.0) license, which permits others to distribute, remix, adapt, build upon this work non-commercially, and license their derivative works on different terms, provided the original work is properly cited, appropriate credit is given, any changes made indicated, and the use is non-commercial. See: http://creativecommons.org/licenses/by-nc/4.0/.

\section{REFERENCES}

1 Siegel RL, Miller KD, Jemal A. Cancer statistics, 2018. CA Cancer J Clin 2018;68:7-30.

2 Mottet N, Bellmunt J, Bolla M, et al. EAU-ESTRO-SIOG guidelines on prostate cancer. Part 1: screening, diagnosis, and local treatment with curative intent. Eur Urol 2017;71:618-29.

3 Louie KS, Seigneurin A, Cathcart P, et al. Do prostate cancer risk models improve the predictive accuracy of PSA screening? A metaanalysis. Ann Oncol 2015;26:848-64.

4 Roobol MJ, van Vugt HA, Loeb S, et al. Prediction of prostate cancer risk: the role of prostate volume and digital rectal examination in the ERSPC risk calculators. Eur Urol 2012;61:577-83.

5 Ankerst DP, Hoefler J, Bock S, et al. Prostate cancer prevention trial risk calculator 2.0 for the prediction of low- vs high-grade prostate cancer. Urology 2014;83:1362-8.

6 Lundon DJ, Kelly BD, Foley R, et al. Prostate cancer risk assessment tools in an unscreened population. World J Urol 2015;33:827-32.

7 Foley RW, Gorman L, Murphy K, et al. 385 the ERSPC risk calculator significantly outperforms the PCPT 2.0 in the prediction of prostate cancer: a multi-institutional study. European Urology Supplements $2016 ; 15$.

8 Foley RW, Lundon DJ, Murphy K, et al. Predicting prostate cancer: analysing the clinical efficacy of prostate cancer risk calculators in a referral population. Ir J Med Sci 2015;184:701-6.

9 Poyet C, Nieboer D, Bhindi B, et al. Prostate cancer risk prediction using the novel versions of the European randomised study for screening of prostate cancer (ERSPC) and prostate cancer prevention trial (PCPT) risk calculators: independent validation and comparison in a contemporary European cohort. BJU Int 2016;117:401-8.

10 Murthy V, Rishi A, Gupta S, et al. Clinical impact of prostate specific antigen (PSA) inter-assay variability on management of prostate cancer. Clin Biochem 2016;49:79-84.

11 Komatsu K, Wehner N, Prestigiacomo AF, et al. Physiologic (intraindividual) variation of serum prostate-specific antigen in 814 men from a screening population. Urology 1996;47:343-6.

12 Morote J, Raventós CX, Lorente JA, et al. Intraindividual variations of total and percent free serum prostatic-specific antigen levels in patients with normal digital rectal examination. Eur Urol 1999;36:111-5.

13 Lavallée LT, Binette A, Witiuk K, et al. Reducing the harm of prostate cancer screening: repeated prostate-specific antigen testing. Mayo Clin Proc 2016;91:17-22.

14 Gómez-Gómez E, Carrasco-Valiente J, Blanca-Pedregosa A, et al. European randomized study of screening for prostate cancer risk calculator: external validation, variability, and clinical significance. Urology 2017;102:85-91.

15 Epstein Jl, Allsbrook WC, Amin MB, et al. The 2005 International Society of Urological Pathology (ISUP) consensus conference on Gleason grading of prostatic carcinoma. Am J Surg Pathol 2005;29:1228-42.
16 Fawcett T. An introduction to ROC analysis. Pattern Recognit Lett 2006;27:861-74.

17 DeLong ER, DeLong DM, Clarke-Pearson DL. Comparing the areas under two or more correlated receiver operating characteristic curves: a nonparametric approach. Biometrics 1988;44:837-45.

18 Zhou X-H, McClish DK, Obuchowski NA, Electronic Book Collection, Wiley InterScience. Statistical methods in diagnostic medicine [Internet]. 2nd edn. Wiley, 2011: 545. https://www.wiley.com/en-us/ Statistical+Methods+in+Diagnostic+Medicine\%2C+2nd+Edition-p9780470183144

19 Hendriksen JMT, Geersing GJ, Moons KGM, et al. Diagnostic and prognostic prediction models. J Thromb Haemost 2013;11 Suppl $1: 129-41$.

20 Vickers AJ, Elkin EB. Decision curve analysis: a novel method for evaluating prediction models. Med Decis Making 2006;26:565-74.

21 Loeb S, Bjurlin MA, Nicholson J, et al. Overdiagnosis and overtreatment of prostate cancer. Eur Urol 2014;65:1046-55.

22 Loeb S, Lilja H, Vickers A. Beyond prostate-specific antigen: utilizing novel strategies to screen men for prostate cancer. Curr Opin Urol 2016;26:459-65-65.

23 Johnston E, Pye H, Bonet-Carne E, et al. INNOVATE: a prospective cohort study combining serum and urinary biomarkers with novel diffusion-weighted magnetic resonance imaging for the prediction and characterization of prostate cancer. BMC Cancer 2016;16:816.

24 McDonald ML, Parsons JK. 4-Kallikrein test and kallikrein markers in prostate cancer screening. Urol Clin North Am 2016;43:39-46.

25 Lepor A, Catalona WJ, Loeb S. The prostate health index: its utility in prostate cancer detection. Urol Clin North Am 2016;43:1-6.

26 De Luca S, Passera R, Cappia S, et al. Fluctuation in prostate cancer gene 3 (PCA3) score in men undergoing first or repeat prostate biopsies. BJU Int 2014;114:E56-61.

27 Ahmed HU, El-Shater Bosaily A, Brown LC, et al. Diagnostic accuracy of multi-parametric MRI and TRUS biopsy in prostate cancer (PROMIS): a paired validating confirmatory study. Lancet 2017;389:815-22.

28 Grill S, Fallah M, Leach RJ, et al. Incorporation of detailed family history from the Swedish family cancer database into the PCPT risk calculator. J Urol 2015;193:460-5.

29 Sölétormos G, Semjonow A, Sibley PEC, et al. Biological variation of total prostate-specific antigen: a survey of published estimates and consequences for clinical practice. Clin Chem 2005;51:1342-51.

30 Nordström T, Adolfsson J, Grönberg H, et al. Repeat prostatespecific antigen tests before prostate biopsy decisions. J Natl Cancer Inst 2016;108. doi:10.1093/jnci/djw165. [Epub ahead of print: 14 Jul 2016].

31 Ankerst DP, Gelfond J, Goros M, et al. Serial percent free prostate specific antigen in combination with prostate specific antigen for population based early detection of prostate cancer. J Urol 2016;196:355-60.

32 Roehrborn CG, Girman CJ, Rhodes T, et al. Correlation between prostate size estimated by digital rectal examination and measured by transrectal ultrasound. Urology 1997;49:548-57.

33 Roehrborn CG, Sech S, Montoya J, et al. Interexaminer reliability and validity of a three-dimensional model to assess prostate volume by digital rectal examination. Urology 2001;57:1087-92.

34 Alberts AR, Schoots IG, Bokhorst LP, et al. Risk-based patient selection for magnetic resonance imaging-targeted prostate biopsy after negative transrectal ultrasound-guided random biopsy avoids unnecessary magnetic resonance imaging scans. Eur Urol 2016;69:1129-34.

35 Chiu PK, Alberts AR, Venderbos LDF, et al. Additional benefit of using a risk-based selection for prostate biopsy: an analysis of biopsy complications in the Rotterdam section of the European randomized study of screening for prostate cancer. BJU Int 2017;120:394-400.

36 Loeb S, Shin SS, Broyles DL, et al. Prostate health index improves multivariable risk prediction of aggressive prostate cancer. BJU Int 2017;120:61-8.

37 Ahmed $\mathrm{HU}, \mathrm{Hu}$ Y, Carter T, et al. Characterizing clinically significant prostate cancer using template prostate mapping biopsy. J Urol 2011;186:458-64. 
Correction: Observational study comparing the accuracy/ variability between the ERSPC and the PCPT risk calculators for the prediction of significant prostate cancer in patients with PSA $<10 \mathrm{ng} / \mathrm{mL}$

Gomez Gomez E, Salamanca Bustos JJ, Carrasco Valiente J, et al. Observational study comparing the accuracy/variability between the ERSPC and the PCPT risk calculators for the prediction of significant prostate cancer in patients with PSA $<10 \mathrm{ng} / \mathrm{mL} \mathrm{BMJ}$ Open 2019;9:e031032. doi: 10.1136/bmjopen-2019-031032

This article was previously published with an error.

The name of the author Raul Luque is incomplete. It should be Raul Miguel Luque.

Raul Miguel Luque is also a co-corresponding author with email address raul.luque@ uco.es.

Open access This is an open access article distributed in accordance with the Creative Commons Attribution Non Commercial (CC BY-NC 4.0) license, which permits others to distribute, remix, adapt, build upon this work non-commercially, and license their derivative works on different terms, provided the original work is properly cited, appropriate credit is given, any changes made indicated, and the use is non-commercial. See: http://creativecommons.org/licenses/by-nc/4.0/.

(C) Author(s) (or their employer(s)) 2020. Re-use permitted under CC BY-NC. No commercial re-use. See rights and permissions. Published by BMJ.

BMJ Open 2020;10:e031032corr1. doi:10.1136/bmjopen-2019-031032corr1

Check for updates 